Disciplinarisation des comportements musulmans dans l'espace public. Pratiques musulmanes et entreprises d'étiquetage à Rennes, Bristol, Nantes et Glasgow

Disciplinary Government of Muslim Conduct in Public Spaces. Muslim Practices and Labelling Processes in Rennes, Bristol, Nantes and Glasgow

\title{
Alice Picard
}

\section{OpenEdition}

Édition électronique

URL : http://journals.openedition.org/rfcb/6868

DOI : $10.4000 /$ rfcb.6868

ISSN : 2429-4373

Éditeur

CRECIB - Centre de recherche et d'études en civilisation britannique

Référence électronique

Alice Picard, «Disciplinarisation des comportements musulmans dans l'espace public. Pratiques musulmanes et entreprises d'étiquetage à Rennes, Bristol, Nantes et Glasgow », Revue Française de Civilisation Britannique [En ligne], XXV-4 | 2020, mis en ligne le 16 octobre 2020, consulté le 24 octobre 2020. URL : http://journals.openedition.org/rfcb/6868 ; DOI : https://doi.org/10.4000/rfcb.6868

Ce document a été généré automatiquement le 24 octobre 2020

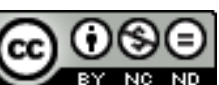

Revue française de civilisation britannique est mis à disposition selon les termes de la licence Creative Commons Attribution - Pas d'Utilisation Commerciale - Pas de Modification 4.0 International. 


\title{
Disciplinarisation des comportements musulmans dans l'espace public. Pratiques musulmanes et entreprises d'étiquetage à Rennes, Bristol, Nantes et Glasgow
}

\author{
Disciplinary Government of Muslim Conduct in Public Spaces. Muslim Practices \\ and Labelling Processes in Rennes, Bristol, Nantes and Glasgow
}

Alice Picard

\section{Introduction}

"See it, say it, sorted». Toute personne un tant soit peu familière des transports en commun britanniques a déjà entendu cette annonce automatique mettant en garde contre les colis piégés déposés dans les gares ou stations de métro et invitant à rapporter la présence de tout bagage suspect à un membre du personnel. Dans cet article, nous proposons d'appréhender cette anecdote comme l'illustration d'un mouvement plus général imputable à l'État britannique qui tend à faire de tout.e citoyen.ne un.e auxiliaire de l'action publique anti-terroriste. Nous invitons à l'envisager de manière parallèle à un autre mouvement, français cette fois-ci, qui se revendique de la laïcité pour mettre en cause certaines pratiques musulmanes dans l'espace public. Qu'il s'agisse de l'exécutif britannique ou de certains pans du secteur associatif laïque, nous souhaitons démontrer ici qu'il est possible de les envisager comme des entrepreneurs symboliques, à l'origine d'une entreprise d'étiquetage qui fait des pratiques musulmanes visibles (prière, alimentation halal, jeûne, port du foulard ou du voile, etc.) des pratiques déviantes et qui s'appuie sur une certaine 
interprétation du sécularisme ou de la laïcitée . En déligitimant l'expression de la religiosité musulmane hors de la sphère privée, cette entreprise contribue à la redéfinition des frontières entre espaces public et privé. Cette reconfiguration se réalise à travers la redéfinition des expressions de la religiosité qui y sont tolérées ou non. Comme le souligne Amélie Barras, les critères qui servent à construire les espaces séculiers évoluent sans arrêt ${ }^{3}$. Cet article a pour objectif d'illustrer le fait que ce "processus disciplinaire " ${ }^{4}$, qui inclut ou exclut en instaurant des conditions d'entrée dans l'espace public est à la fois le produit de l'action des concepteurs/trices des politiques publiques et des citoyen.ne.s qui se sentent concerné.e.s. La prévention de la radicalisation au Royaume-Uni joue ce rôle de disciplinarisation des comportements dans l'espace public. Ce processus de disciplinarisation se déploie au travers de mesures exceptionnelles qui aboutissent au traitement différencié de l'islam par rapport aux autres religions et des musulman.e.s par rapport à d'autres minorités. L'entreprise symbolique analysée ici constitue l'un des aspects de la construction de l'islam en problème public en France et au Royaume-Uni qui doit elle-même être rapportée à la construction progressive de la religion musulmane en tant que catégorie d'action publique. Cette problématisation ne passe pas seulement par la redéfinition des expressions de la visibilité religieuse acceptables, elle s'appuie également sur la lutte contre le terrorisme et la radicalisation. De part et d'autre de la Manche, les législations antiterroristes existaient bien avant les diverses attaques perpétrées par de jeunes hommes musulmans et attribuées à leur radicalisation religieuse. Néanmoins, les attentats commis sur les sols français et britannique, en 2005 à Londres, par deux fois en 2015 à Paris, en 2016 à Nice et à plusieurs reprises en 2017 à Manchester puis à Londres ont contribué à la mise en équivalence entre islam et violence politique (l'équation «Muslims equal terrorists »), qui se construit par ailleurs à l'échelle internationale depuis les attentats du World Trade Center le 11 septembre 2001, et à l'opposition binaire entre "islam modéré » et «islam radical». Or, il apparaît que l'« islam modéré » est aussi le plus sécularisé et que les signes d'islamité sont associés à l'intégrisme ${ }^{6}$.

2 Nous empruntons, pour ce faire, les concepts d'étiquetage à Howard Becker ${ }^{7}$ et de status movement à Joseph Gusfield ${ }^{8}$. Becker et Gusfield, qui étudient respectivement les fumeurs de marijuana et le mouvement de tempérance (temperance movement), s'inscrivent tous deux dans une perspective interactionniste. Ils désignent les formes d'action collective dont l'objectif est de transformer un acte perçu comme déviant en action illégale par les termes d'entreprise ou de croisade morale ${ }^{9}$ ou symbolique. Il n'y a pas de déviance sans processus d'étiquetage, c'est-à-dire sans jugement social vis-à-vis d'un comportement dont les entrepreneur.e.s de morale estiment qu'il enfreint les valeurs et les normes que s'est données un groupe social particulier. Cette forme d'action collective est une entreprise normative.

3 Cet article rend compte d'une partie des résultats d'une recherche doctorale, dont l'enquête a été réalisée entre octobre 2015 et juin 2018 au Royaume-Uni et en France. La démarche comparative a mis en regard deux municipalités britanniques (Bristol et Glasgow) et deux municipalités françaises (Rennes et Nantes) en matière de gouvernance du fait musulman. Bristol et Rennes ont constitué les cas d'étude principaux tandis que, pour éviter toute montée en généralité abusive, Nantes et Glasgow ont servi de cas contrôles. La problématique choisie visait l'exploration de deux dimensions de l'objet: d'une part l'influence de dits modèles nationaux qui 
concernent les liens entre État et Églises sur les relations entre puissance publique (y compris locale) et populations musulmanes et d'autre part la problématisation de la visibilisation de la présence musulmane dans deux sociétés qui se perçoivent comme sécularisées et la reconfiguration des frontières symboliques entre public et privé mais aussi entre national et étranger.

4 La recherche s'est appuyée sur des méthodes qualitatives. Soixante-quinze entretiens semi-directifs ont été réalisés auprès d'élu.e.s et d'employé.e.s des municipalités étudiées, d'acteurs et d'actrices du monde associatif musulman (culturel et cultuel) mais aussi du secteur associatif relevant des race relations et des equalities (lutte contre les discriminations) au Royaume-Uni et relevant de la défense ou de la promotion de la laïcité en France. Ces entretiens ont été complétés par la consultation des archives municipales et plus précisément des comptes-rendus des délibérations des conseils municipaux et des commissions bristoliennes travaillant successivement sur la « race » pour calquer la terminologie en vigueur oure-Manche, la lutte contre les discriminations et la community cohesion ("cohésion sociale»). La presse locale a également été explorée, sur des périodes identiques (1989-1993 ; 1997-2002 ; 2003-2005 et 2012-2017), afin d'englober, en sus de la période la plus récente, des dates charnières dans la construction de l'islam en problème public ; à savoir 1989 et 2001. Cette problématisation de la religion et des pratiques musulmanes mène à une focalisation de l'attention publique sur elles et à leur exceptionnalisation, qui appelle un traitement dérogatoire du droit ou des coutumes communes. Enfin, l'enquête a consisté en quelques observations participantes, essentiellement à Rennes et à Bristol. À Rennes, ces observations ont concerné à la fois des réunions publiques organisées à l'occasion de l'anniversaire de la loi de séparation des Églises et de l'État (9 décembre 1905) mais également des sessions de formation sur la thématique de la laïcité destinées pour une partie aux animateurs et animatrices d'ateliers qui s'adressaient à des publics scolaires et pour l'autre aux agent.e.s de la collectivité intervenant dans les écoles primaires. À Bristol, les séquences d'observation ont inclus notamment des sessions de formation à Prevent, un des piliers des diverses législations antiterroristes britanniques des dernières années, destinées encore une fois aux employé.e.s de la fonction publique territoriale et aux bénévoles du secteur associatif. À Nantes, il s'est agi, de manière très ponctuelle, d'une session de formation à la laïcité, organisée par la Ligue de l'enseignement sur deux jours et destinées à des fonctionnaires territoriaux ou à des bénévoles du secteur associatif.

5 L'article aborde l'entreprise symbolique d'étiquetage de la visibilité religieuse musulmane en deux temps. Tout d'abord, il s'agit de montrer qu'à Nantes et Rennes, une partie du mouvement laïque endosse localement une controverse nationale qui s'articule autour des définitions concurrentes de la laïcité. L'entreprise est également normative en ce qu'elle se donne pour mission d'établir quelles expressions de la religiosité musulmane dans l'espace public contreviennent au principe laïque. Ensuite, il s'agit de s'intéresser aux cas britanniques où l'État entreprend de revenir sur l'autonomie octroyée aux collectivités locales dans le cadre de sa politique antiterroriste. Il multiplie ainsi ses auxiliaires et leur laisse peu de marge de manœuvre dans leur interprétation des comportements qui signalent un processus de radicalisation. 


\section{Luttes définitionnelles autour de la laïcité : une entreprise axiologique autant que normative}

6 Pour comprendre le contexte dans lequel agit le mouvement laïque, il est nécessaire de rappeler que l'année 2015 a permis de mettre la religion musulmane à l'agenda local à Nantes et à Rennes sous couvert de laïcité. Le choc provoqué par les attentats du mois de janvier 2015, et plus encore ceux du mois de novembre, a poussé les municipalités rennaise et nantaise à réagir. Les politiques antiterroristes et de renseignements ne relèvent toutefois pas de leurs prérogatives. La laïcité est alors apparue comme un instrument symbolique de protection de la République dont elles pouvaient se saisir. Quelques dispositifs ont contribué à transformer l'islam en objet de discussion publique : la mise sur pied d'un Comité consultatif laïcité à Rennes et l'organisation d'un cycle de rencontres sur le même thème à Nantes ainsi que des formations à destination des agent.e.s publics et des professionnel.le.s du secteur socio-culturel. Cette politisation soudaine de l'islam au travers de la laïcité est d'autant plus étonnante que ni l'un ni l'autre sujet n'étaient à l'agenda des municipalités et en tous les cas, pas de manière problématique. Elle a été éphémère à Nantes où la municipalité n'a pas pris d'autres initiatives au-delà de l'année 2016 et elle était sans doute quelque peu antérieure à Rennes où une partie du mouvement laïque contestait depuis longtemps le financement municipal d'un certain nombre de centres culturels servant de facto de lieux de culte ${ }^{10}$. La mise en discussion de la laïcité dans l'espace public a validé symboliquement l'entreprise d'une partie du mouvement laïque. Celui-ci doit en effet être appréhendé de manière dynamique et hétérogène. Plusieurs conceptions de la laïcité s'affrontent en effet en son $\operatorname{sein}^{11}$. Ces conceptions n'empruntent néanmoins qu'aux formes historiques de la laïcité, telles qu'elles ont été distinguées par Jean Baubérot dans sa typologie. Lles conceptions antireligieuse, séparatiste-individualiste et séparatiste-collective mènent à des désaccords entre pans du mouvement laïque. À Rennes et à Nantes, ce mouvement s'est institutionnalisé entre le $\mathrm{XIX}^{\mathrm{e}}$ et le $\mathrm{XX}^{\mathrm{e}}$ siècles, autour du conflit qui a opposé l'Église catholique aux Républicain.e.s et qui portait sur le contrôle de la sphère publique. Les anti-cléricaux luttaient contre l'emprise des institutions religieuses sur les affaires publiques et a abouti tout d'abord à l'instauration, par les lois Ferry au début des années 1880, d'une école publique, gratuite et laïque puis à la séparation des Églises et de l'État par la loi du 9 décembre 1905. Le mouvement laïque a peu à peu été associé aux politiques publiques municipales des deux villes et constitue encore aujourd'hui un acteur essentiel des réseaux de gouvernance municipaux du fait de la présence de majorités socialistes depuis plusieurs dizaines d'années. Dans le cadre de la mise à l'agenda local de l'islam et des pratiques musulmanes, ce mouvement met en jeu sa qualité d'auxiliaire de l'action publique municipale en matière de régulation du fait religieux. Ceci étant dit, ce sont surtout les tenant.e.s de la nouvelle laïcité qui participent de la disciplinarisation des pratiques religieuses dans l'espace public. Il ne s'agit pas ici d'analyser une entreprise anti-musulmane mais de problématisation et d'exceptionnalisation de certains signes d'islamité. 


\section{Mettre en discussion la laïcité pour mieux parler d'islam}

7 Les mouvements laïques dont il est question ici sont à envisager comme des mouvements sociaux ${ }^{12}$. Comme d'autres, ils sont également caractérisés par le désir d'être reconnus par les pouvoirs publics comme interlocuteurs légitimes à propos du sujet autour duquel ils se mobilisent. Il s'agit toutefois de réseaux quelque peu différents à Rennes et à Nantes. Dans la première ville, mentionnons le Comité Laïcité 35 qui se présente comme une coalition hétéroclite dans laquelle se rassemble une bonne partie des associations laïques issues de l'Éducations nationale (notamment les syndicats) ou proches de celle-ci, des associations d'éducation populaire ainsi que des associations entièrement dédiées à la promotion ou la défense de la laïcité. Les conceptions de la laïcité de ces différentes associations apparaissent parfois inconciliables et elles sont inégalement dotées pour les faire valoir. Si les plus associations les plus antireligieuses s'appuient sur le cadre collectif pour déployer leurs actions d'interpellation ou de sensibilisation, d'autres structures, davantage libérales, ont des liens plus directs avec la municipalité ${ }^{13}$. Quant à Nantes, de nombreuses associations du mouvement laïque figuraient au programme du cycle de conférences sur la laïcité, c'est-à-dire la Fédération des associations laïques (FAL) 44, le Centre interculturel de documentation (CID), l'Accoord (centres sociaux) et les Francas.

En tant que status movement, certaines de ces associations cherchent à maintenir le statu quo, c'est-à-dire à demeurer les interlocuteurs légitimes des municipalités en matière de prise en charge de la jeunesse et de la politique de la ville. Par ailleurs, à travers la mise à l'agenda local de la laïcité, de nombreuses associations cherchent à raviver le débat des rapports entre pouvoirs publics et Église catholique qui se poursuit notamment à travers l'enjeu non négligeable du financement public des établissements scolaires privés. Néanmoins, cette mise en discussion de la laïcité, du fait d'un contexte national propice à la problématisation de l'islam et des pratiques musulmanes, d'autant plus depuis 2015, se transforme de facto en redéfinition des frontières des espaces public et privé et des expressions acceptables de la religiosité.

Ce status movement s'inscrit dans ce qu'Anne-Sophie Lamine identifie comme un premier niveau de conflictualité liée à la pluralité religieuse. Il concerne la régulation publique du religieux, y compris à l'échelle d'une commune ${ }^{14}$. En matière d'islam, la conflictualité se structure autour de l'infraction, par certains signes d'islamité, de valeurs et de normes collectives, dont la liste est sans fin selon Françoise Lorcerie ${ }^{15}$. Le statut social de celles et ceux qui participent au status movement, serait rétabli par le respect des normes sociales qu'un pan des mouvements laïques se donne pour mission de réaffirmer. En matière de laïcité, ce sont les normes séculières et la neutralité qui sont au cœur de la conflictualité. Or, une norme est rarement mieux établie symboliquement que par la voie législative, en particulier en France. Les normes ainsi réaffirmées et même élaborées, bien qu'elles se réfèrent à des principes généraux, sont parfois le support de mesures d'exception qui visent l'islam en particulier.

10 Les pratiques musulmanes sont ainsi mises en discussion publiquement à l'aune des normes et des valeurs qu'elles enfreignent. Ceci participe d'un processus d'étiquetage qui peut être illustré par un exemple issu d'observations de terrain à Rennes. Une formation organisée dans le cadre de la Semaine de la solidarité internationale (SSI) s'est ainsi déroulée en octobre 2016. Le lien entre cet événement porté par les associations de solidarité internationale de la ville de Rennes et la laïcité passe par la 
Maison Internationale de Rennes (MIR) qui a récemment rejoint le Comité Laïcité 35, qui est membre du Comité consultatif laïcité de la ville de Rennes depuis ses débuts et coordonne la SSI (aujourd'hui Festival des solidarités). Dans ce cadre, la MIR souhaite proposer des animations à destination des publics scolaires. Pour cela, elle met sur pied une petite équipe de bénévoles qui a vocation à accueillir des classes dans les locaux de la MIR pour les sensibiliser à la laïcité. L'équipe est composée de membres du Comité Laïcité 35 mais aussi d'associations telles que Coexister ou le CRIDEV (Centre de ressources et d'interpellation pour un monde sans rapports de domination). Les interprétations de la laïcité qui cohabitent au sein de cette équipe sont donc multiples voire antagonistes, d'autant plus que les deux bénévoles de Coexister sont elles-mêmes de confession musulmane et que l'une d'elles porte un hijab. Une formation qui précède les animations se déroule en deux temps et en soirée et est animée par une enseignante, formatrice "Valeurs de la République et laïcité». L'observation participante à cette formation a permis de consigner les différentes réactions des participant.e.s face à la présentation de différents cas pratiques. Les comportements qui relèvent des pratiques musulmanes sont ainsi systématiquement mis en regard de normes sociales dont l'infraction constitue la déviance. Il peut s'agir de la laïcité, du programme scolaire, du droit du travail, d'une loi ou d'une circulaire ou du règlement intérieur. Bien que la formatrice ait pris le soin de sélectionner des cas pratiques qui renvoient à des comportements rituels issus de diverses religions, les commentaires des participant.e.s se focalisent bien souvent sur l'islam. Face au cas d'un élève fortement anémié qui ne parvient pas à se concentrer sur les activités scolaires proposées, et qui dans la réalité est Témoin de Jéhovah, un des participant.e.s déclare que ce cas lui fait penser à une situation à laquelle il a bel et bien été confronté. En tant qu'animateur de colonie de vacances, il a un jour refusé l'inscription d'un jeune homme qui souhaitait faire le ramadan pour des raisons de sécurité. La formatrice ajoute qu'une telle décision peut facilement être justifiée si le règlement intérieur de la structure mentionne la laïcité. La mise en visibilité de certaines pratiques religieuses est décriée dans chacun des autres cas abordés ${ }^{16}$. La mise en discussion de pratiques essentiellement musulmanes aboutit à l'émergence d'une nouvelle norme collective de gestion des signes extérieurs d'islamité qui fait appel à une certaine interprétation de la laïcité qui privilégie la neutralité et traite l'islam de manière dérogatoire malgré l'appui sur un principe général.

\section{Sphère publique, espace privé et tiers espace : l'extension de la neutralité}

11 L'enjeu de la conflictualité autour de l'islam, lorsqu'elle mobilise la norme laïque, tient dans la définition des frontières séculières généralement établies entre espace public et espace privé. Ces frontières, mouvantes, sont le résultat de luttes définitionnelles qui opposent schématiquement les associations qui placent la focale sur la neutralité, en imputant la responsabilité du problème aux croyant.e.s dont les comportements ne respectent pas le principe de laïcité à celles qui la placent sur la séparation, en imputant le problème à l'État. C'est la première approche qui tend à s'imposer ces dernières années à l'échelle nationale. La séparation est donc marginalisée au profit de la neutralité ${ }^{17}$. Valérie Amiraux identifie également cette tendance: «Depuis plus de dix ans, la charge de la neutralité, pilier de laïcité, s'est [...] déplacée des institutions publiques de l'État vers les individus $»^{18}$. L'appréciation de la neutralité s'est étendue au 
cours des dernières années par plusieurs biais. Le premier consiste à complexifier la distinction entre sphère privée et sphère publique. La sphère publique a pendant longtemps fait référence à la seule fonction publique. Selon cette approche, l'espace public appartient à la sphère privée en matière d'expression de la religiosité. Or, le Haut conseil à l'intégration (HCI) introduit une nouvelle notion à partir de 2010-2011 : celle d'espace social ${ }^{19}$ où la laïcité, et en réalité la neutralité, pourrait devoir s'appliquer. À mesure que les frontières des espaces sont redéfinies, les espaces où est exigée une certaine retenue dans l'expression de la religiosité des individus s'étendent. Cette extension de la neutralité dans l'espace crée de nouvelles infractions à la laïcité qui multiplient les situations d'exception entre religions dont la visibilité est rendue problématique et celles, telles les confessions chrétiennes, dont la pratique séculaire a influencé notamment l'organisation des temps sociaux.

On trouve un exemple de complexification de la notion d'espace à Rennes, dans le rapport du Comité consultatif laïcité. Celui-ci va même jusqu'à distinguer quatre espaces :

Les règles découlant du principe de laïcité ne s'appliquent pas de la même manière selon que l'on est dans l'espace public ou dans l'espace privé. Mais pour être plus précis encore il conviendrait de distinguer quatre espaces, bien que cette distinction ne soit pas toujours évidente : L'espace privé : c'est l'espace où l'on est totalement libre, sous la seule réserve du respect de la loi. L'espace administratif: c'est l'espace de l'état, des collectivités locales, des services publics. [...]. L'espace social: c'est l'espace où l'on travaille ensemble, l'association par exemple ou l'entreprise. Là, la liberté de conscience est garantie, sous réserve d'absence de prosélytisme, du respect des règles d'hygiène et de sécurité, mais aussi du respect de la bonne marche de l'entreprise. L'espace partagé: c'est l'espace de la communauté de ville, la rue par exemple ou la place publique, qui ne saurait être confondu avec l'espace administratif. Là, la liberté de conscience est garantie, y compris pour une expression du religieux, dans la limite de l'interêt de l'ordre public ${ }^{20}$.

Il est surprenant qu'il ne soit pas rappelé que la laïcité ne s'applique pas aux espaces public et privé mais bien à la sphère publique, désignée ici par espace administratif. L'extrait ci-dessus révèle une opération de publicisation de l'espace privé qui passe par l'élaboration d'un espace social et d'un espace partagé auxquels s'applique la neutralité. Le même rapport, même s'il adopte des formulations générales, est émaillé de références aux pratiques musulmanes problématisées dans le cadre des activités associatives ou des liens entre agent.e.s de la municipalité et usager.e.s musulman.e.s. Les attitudes à adopter face à la prière rituelle musulmane, au jeûne, au port du foulard ou du voile ou aux repas sans porc dans les cantines constituent la majorité des exemples et des préconisations du rapport.

Le deuxième biais par lequel s'étend la neutralité est celui de la substantialisation ou de l'axiologisation de la laïcité. Dans les discours, cette dernière devient de plus en plus dans les discours une valeur et non plus un principe d'action publique. Par le passage de la neutralité-séparation à la neutralité-laïcisation ${ }^{21}$, la laïcité acquiert un contenu substantiel qui s'applique aux personnes privées. Elle invite à s'intéresser autant aux comportements individuels qu'aux symboles religieux et laisse ainsi davantage de place à la subjectivité des interprétations. De fait, sur les terrains nantais et rennais, la laïcité oscille très souvent entre valeur et principe. Les mêmes personnes ou organisations l'utilisent tour à tour dans un sens ou dans l'autre. Ainsi le maire de Nantes, Johanna Rolland, est citée, le 9 janvier 2015, c'est-à-dire au lendemain des attaques contre la 
rédaction de Charlie Hebdo et l'hypercasher, par Ouest-France de la façon suivante : " "La République, a-t-elle rappelé, ce sont des valeurs : liberté, égalité, fraternité, laïcité. C'est un combat de tous les jours pour que ces mots si forts, soient chaque jour plus réels, plus concrets. La République, c'est ce qui nous rassemble. Ce ne sont pas les amalgames. La République, c'est le service public, notre bien commun, qui agit, qui protège et est souvent le dernier rempart pour les plus précaires" ${ }^{22}$. Peut-être les propos du maire sont-ils déformés. Toutefois l'erreur est rendue possible du fait qu'il existe une certaine confusion entre valeur et principe.

La publicisation des espaces sociaux et l'axiologisation de la laïcité se traduisent déjà par des lois, des jurisprudences et des chartes auxquelles les mouvements laïques, et notamment les tenant.e.s de la nouvelle laïcité, peuvent se référer pour justifier leur action et valider leurs prescriptions. Ainsi lors de la deuxième séance de la formation proposée par la MIR dans le cadre de la SSI 2016, une militante d'ÉGALE rappelle la « lettre de la loi» du 15 mars 2004 après qu'une autre participante a mentionné l'obligation d'accueil des enseignant.e.s à propos du port du hijab par des élèves. Alors qu'un débat s'engage sur ce qui est ostentatoire ${ }^{23}$ ou ne l'est pas, elle tente d'y couper court en affirmant que «la loi, c'est la loi $»^{24}$. Ce qui traduit bien ici le fait que la législation est favorable à ses jugements sociaux vis-à-vis du fait religieux. Or, l'axiologisation de la laïcité fait constamment évoluer la norme légale à laquelle les mouvements laïques se réfèrent pour se légitimer symboliquement. Cette évolution normative continue tend à faire de l'exception une règle plus difficilement contestée ou discutée.

\section{Mise à l'agenda endogène ou répliquée?}

16 La mise à l'agenda local de la laïcité, qui dépasse les seuls conseils municipaux et concerne leurs réseaux de manière générale, peut apparaitre comme une réplique localisée de la focalisation sur l'islam à l'échelle nationale. Des indices dessinent toutefois les contours d'un processus de problématisation endogène sans qu'il soit possible de trancher entre une émergence spontanée ou suscitée. Quoi qu'il en soit, les professionnel.le.s en relation avec les publics municipaux - agent.e.s de guichet, professionnel.le.s du secteur socio-culturel ou du secteur éducatif, semblent exprimer une demande de normes. La frilosité d'une partie de la fonction publique municipale et du secteur socio-éducatif à endosser la responsabilité de décisions prohibitionnistes ou libérales dans un contexte de discussions publiques intenses rappelle le "désarroi " décrit par Françoise Lorcerie dans le cadre de son étude de la mobilisation des chef.fe.s d'établissement en 2003-200425. Les professionnel.le.s peuvent se sentir exposée.e.s lorsque la jurisprudence est contradictoire (donc au cas par cas) car elle fait reposer sur elles et eux l'appréciation subjective des comportements des usager.e.s.

Il faut prendre les indices recueillis avec toutes les précautions qui s'imposent en matière de documents produits par les municipalités ou par les réseaux qui participent aux politiques publiques municipales qui visent en partie à légitimer les choix municipaux. Néanmoins, on peut citer à titre d'exemple l'une des versions du "Référentiel commun autour de l'application du principe de laïcité sur les temps péri et extra scolaires de la Ville de Rennes", lequel indique que "les professionnels ont également exprimé l'intérêt de trouver des points de repères dans les décisions politiques: les élus pourraient ainsi faire part de leur avis concernant l'utilisation de certains vocabulaires ${ }^{26}$. Cette remarque intervient au cours d'une partie consacrée à la laïcité et aux temps de 
restauration. Le référentiel en question est le résultat d'une formation action que la Ville de Rennes a confiée à la Ligue de l'enseignement dans le cadre de la révision du projet éducatif local et que cette dernière a proposé à la Direction Enfance Éducation de la municipalité. À Nantes, le constat est identique et une démarche similaire a été entreprise, sans implication directe la municipalité mais avec son soutien toutefois. Le Centre interculturel de documentation (CID) a été à l'origine d'un travail qui a rassemblé des organisations du secteur associatif de Loire-Atlantique et qui a donné lieu à la publication, en 2016, d'une brochure intitulée La laïcité au quotidien. Repères et situations de terrain. Interrogée quant à son ressenti en matière d'attentes de la part des professionnel.le.s et des bénévoles, Asma B, salariée du CID, répond de la façon suivante :

Oui parce que beaucoup de professionnels demandent aussi d'avoir des clés de compréhension. Nous avons fait tout un travail avec Dounia Bouzar, justement et des professionnels de l'animation sociale et culturelle, des éducateurs, des directeurs de Maisons de Quartier. Et ça a donné lieu à ce référentiel justement sur les postures professionnelles. Et il y a des professionnels qui se retrouvent en grande difficulté quand ils sont en face à face avec quelqu'un de culture et de tradition musulmane. Et qui parfois ont un discours ou une posture vis-à-vis de la femme ou sur la question du voile, et comment on accueille, et comment on répond sans être taxé de raciste ou être accusé de faire de la discrimination. C'est vrai que c'est compliqué d'avoir une posture professionnelle objective quand on ne connaît pas la culture de l'autre ${ }^{27}$.

Les agent.e.s de ces deux collectivités attendent ainsi des élu.e.s des directives claires pour éviter d'être accusé.e.s de discrimination ou de racisme et se prémunir contre toute contestation de la part des usager.e.s des services publics municipaux. Ainsi, si l'entreprise de politisation de la laïcité et de l'islam à Rennes et à Nantes ne trouve pas sa source parmi ces agent.e.s, elle y trouve un relais.

\section{Lutte contre la radicalisation et gouvernement des comportements musulmans}

Tandis qu'une entreprise symbolique a été décrite pour analyser les dynamiques actuelles au sein des mouvements laïques à Rennes et à Nantes, c'est davantage le retour de l'État au Royaume-Uni qui doit être exploré dans cette seconde partie. Localement, les pouvoirs publics et leurs agent.e.s sont tenu.e.s d'appliquer les directives du gouvernement central. La construction d'une déviance nouvelle qui se donne pour objet de réforme l'orthopraxie et l'orthodoxie musulmanes ainsi qu'une nouvelle forme de gouvernementalité ${ }^{28}$ des corps musulmans dans l'espace public sont les deux éléments qui permettent de tisser des liens entre les cas des deux côtés de la Manche. Ces liens ne doivent toutefois pas faire oublier que le port de signes religieux visibles est bien davantage admis au Royaume-Uni où il est accepté y compris de la part des fonctionnaires, exception faite du port du voile intégral. Depuis 2011, à la suite de l'accession au pouvoir d'une nouvelle majorité conservatrice, le gouvernement britannique a procédé à des modifications de législation, ce qui lui permet de reprendre la main et de ne pas compter sur l'intermédiation des collectivités locales et de leurs réseaux de gouvernance pour mettre en œuvre son programme de lutte contre la radicalisation. Les fonds du programme Prevent ont ainsi été retirés à un certain nombre de municipalités qui y avaient jusqu'alors droit. Hors des territoires définis 
comme des cibles $^{29}$, et qui bénéficient encore de ces fonds, le gouvernement recourt à un mode de gestion bien plus direct et centralisé. Il propose un programme de formations qui visent à donner aux agent.e.s de l'État et des collectivités locales, ainsi qu'aux auxiliaires de l'action publique, les outils pour repérer les signes de la radicalisation chez les publics que ces professionnel.le.s prennent en charge. La particularité de ces formations est que leur contenu est fourni clé en main par le Home Office. De cette façon, le gouvernement s'assure que les marges d'interprétation de sa politique par les collectivités locales et les organismes publics sont réduites au minimum. Avant 2011, l'allocation des fonds Prevent était décidée, à Bristol, en concertation avec les communautés musulmanes, au sein d'une instance consultative nommée Building the Bridge, qui a servi à institutionnaliser les réseaux de gouvernance musulmans. Néanmoins, ette forme de community ownership ${ }^{30}$ (contrôle citoyen) n'empêchait pas la forte contestation suscitée par Prevent au sein des populations musulmanes. Un rapport publié en 2009 présentait ainsi le programme comme un dispositif d'espionnage et dénonçait les critères d'allocation des fonds ${ }^{31}$. Ces derniers apparaissaient tout à la fois théologiques (distinction contestable entre modérés et radicaux) et politiques (exclusion des associations critiques du gouvernement du dispositif). La construction d'une "communauté suspecte» (suspect community) musulmane est également dénoncée puisqu'elle en fait une communauté qui doit être particulièrement surveillée. La notion de "communauté suspecte» a d'abord été appliquée aux populations irlandaises soupçonnées de soutenir la violence politique de l'organisation indépendantiste Irish Republican Army (IRA). Les différentes générations de législations anti-terroristes instaurent donc chacune à leur manière une forme de gouvernement de l'exception qui cible certaines populations en particulier. En 2011, la mise à disposition des fonds Prevent cesse. La transformation du programme de lutte contre la radicalisation a ainsi contribué à déposséder les communautés musulmanes des politiques publiques qui s'appliquent pourtant en priorité à elles.

\section{Recentralisation et ambition formatrice}

20 Avant 2011, il était admis qu'une large part des fonds mis à disposition par le programme Prevent était en réalité utilisée par les autorités locales pour soutenir des activités relevant de la community cohesion et des projets communautaires ${ }^{32}$. Le référentiel de la community cohesion s'impose au début des années 2000 à la suite de la publication d'une série de rapports consacrés aux révoltes urbaines des villes d'Oldham, Bradford ou Burnley au cours de l'été 2001. Ces révoltes, qui impliquent des jeunes hommes d'origine Asian, sont présentées comme le résultat de décennies de politiques différentialistes qui ont permis que soient menées des « vies parallèles ». La notion de community cohesion apparaît dans le rapport Cantle, commandité par David Blunkett, alors Home Secretary. Elle est suffisamment floue pour donner lieu à des interprétations aussi bien multiculturalistes qu'intégrationnistes. Elle promeut l'adhésion à des valeurs communes (shared values), le renforcement des liens sociaux entre communautés et un sentiment d'appartenance commune à la communauté nationale ${ }^{33}$. Du fait de la recentralisation de la gouvernance de Prevent et de la plus stricte distinction entre community cohesion et dimension sécuritaire au sein de la nomenclature ministérielle ${ }^{34}$, cette dernière dimension a pris de l'importance. Entre 2007 et 2011, sous un gouvernement travailliste, le programme Prevent avait alloué des fonds aux collectivités locales sur la seule base du nombre de musulman.e.s résidant sur 
leur territoire. L'utilisation des fonds était globalement assez peu contrôlée. Bristol s'en est servi pour financer des projets associatifs portés par des organisations musulmanes entre 2007 et 2011 et notamment par les mosquées. Le Home Office préfère désormais employer de façon directe des chargé.e.s de mission Prevent (Prevent coordinators) et financer la tenue de multiples formations destinées aux agent.e.s de guichet (frontline personnel) des organismes et administrations publiques. La version 2018 du programme CONTEST, dans lequel Prevent s'insère mais qui a un objectif plus large de lutte contre le terrorisme, indique : «Prevent training has been completed over one million times ${ }^{35}$.

21 Ces formations sont non seulement le résultat d'une évolution du programme Prevent à partir de 2011 mais s'insèrent également dans le Prevent Duty, inscrit dans le Terrorism and Security Act qui date de 2015, et qui définit les devoirs qui s'imposent légalement aux agent.e.s du secteur public en matière de participation à la lutte contre la radicalisation et à la protection des publics définis comme vulnérables. La loi intéresse plusieurs autorités et institutions spécifiques et exige : «due regard to the need to prevent people from being drawn into terrorism $»^{36}:$ "The duty requires local authorities, schools, colleges, higher education institutions, health bodies, prisons and probation, and the police to consider the need to safeguard people from being drawn into terrorism ». Plusieurs types de formation existent qui visent à sensibiliser les agent.e.s et les bénévoles du secteur associatif à ces devoirs. Les plus connues sont conçues par le gouvernement lui-même et sont dispensées selon un schéma tout à fait formel qui laisse peu, voire pas d'autonomie, aux formateurs/trices. Il s'agit des ateliers WRAP (Workshops to Raise Awareness about Prevent). L'objectif du Home Office est le suivant : " provide Prevent training to a further one million people and work to increase the number of referrals that come from communities and friends and family ${ }^{37}$. Cette réforme du programme Prevent rend son application en Écosse complexe et suscite la contestation d'organisations syndicales qui se sont rassemblées, avec d'autres organisations du secteur associatif, au sein d'un collectif, Scotland Against Criminalising Communities (SACC), actif à Glasgow et Edinburgh principalement. En effet, Prevent s'appuie désormais non plus seulement sur les administrations de sécurité et de renseignement mais également sur la totalité des services publics en contact avec le public desquels il est exigé qu'ils participent au travail de détection des signes de radicalisation. Les organisations syndicales font à cet égard remarquer :

The Prevent strategy is part of the UK's counter-terrorism strategy and is therefore reserved to Westminster, but it is implemented in Scotland through Scottish public bodies (e.g. local authorities, schools, [Higher Education] and [Further Education] colleges, the NHS, Police Scotland, the prison service) and so mainly involves functions that are devolved to Holyrood ${ }^{38}$.

Les ateliers WRAP consistent en des sessions d'une heure seulement organisées autour de l'utilisation de vidéos fournies par le gouvernement qui abordent deux types de radicalisation: "islamiste " et d'extrême droite. Leur contenu est fictionnel. Des personnages types sont mis en scène en guise d'illustration des trajectoires de radicalisation et des raisons qui président à ces évolutions. Lors d'une session à laquelle nous avons pu assister, la première vidéo aborde la question de la radicalisation à l'extrême droite. Il est ensuite demandé aux participant.e.s de travailler en groupe et d'identifier à partir de ces témoignages des facteurs de radicalisation. Les facteurs proposés ce jour-là sont : la colère, la solitude, les pertes d'emploi et le chômage, le manque d'information, des relations sociales précaires, de mauvaises expériences personnelles ou de proches, les problèmes de santé mentale, la prison, les stéréotypes 
véhiculés par les médias et l'isolement culturel. Le formateur ce jour-là commente sobrement : "According to the government, you all did well " ${ }^{39}$. Il a été possible de rencontrer la personne qui dispensait la formation ce jour-là. Il s'agit du salarié d'une organisation répondant au nom d'Integrate UK. Interrogé à propos du temps consacré à la formation, il répond:

I think the time is enough. I think, the content and the way the content is delivered can mean that it does not feel like the time has been used wisely. So at the beginning I faced the claim that I'm reading off a script because most people would assume, they would look at me reading or looking at the person delivering or reading and thinking why are they not delivering as if it's something they do all the time? With WRAP, there's no scope, there's no flexibility at all. We have to stick to that script word for word. Which can sometimes come out as quite robotic. And [...] it feels like the time is not used as well as it should. Because the trainees only get one side of the information ${ }^{40}$.

Saqib V. reconnaît ainsi que le Home Office, qui finance certaines organisations pour dispenser les formations, ne leur laisse aucune marge de manœuvre quant au contenu de la formation et aux réponses que les formateurs/trices peuvent apporter aux participant.e.s. Il s'agit bien là d'un indice de la volonté du gouvernement de reprendre la main sur le programme de lutte contre la radicalisation, à la fois à travers les ateliers WRAP et par l'imposition de devoirs aux agent.e.s du secteur public.

\section{Sécuritarisation de l'action publique}

Pour Claire Arènes, Prevent relève de deux logiques : d'une part une dépolitisation du terrorisme par la focalisation sur les trajectoires individuelles de radicalisation et leur pathologisation et d'autre part une sécuritarisation du soin et des vulnérabilités individuelles et collectives ${ }^{41}$. La multiplication des auxiliaires de l'action étatique et l'enrôlement de l'ensemble des agent.e.s de la fonction publique par le gouvernement dans le cadre de sa politique de lutte anti-terroriste s'inscrit dans ces deux logiques. Cette politique participe par ailleurs, d'un processus d'étiquetage qui construit les comportements musulmans orthopraxes en actes déviants.

Le Prevent Duty met, à ce titre, les agent.e.s de la puissance publique en responsabilité de repérer les signes de radicalisation et de les signaler. Ce que cette évolution législative implique en matière de relation entre gouvernant.e.s et gouverné.e.s mérite d'être souligné. Elle instaure en effet une forme de surveillance au guichet et fait des street level bureaucrats des auxiliaires de la politique de lutte contre la radicalisation alors qu'il ne s'agit pas nécessairement de leur mission première. La sociologie de la street level bureaucracy s'intéresse en particulier à l'autonomie inhérente au travail de guichet. Celle-ci contribue à expliquer les écarts entre les objectifs assignés à une politique publique lors de son élaboration et ses résultats concrets. C'est sans doute pour réduire cet écart que le gouvernement multiplie les formations (WRAP trainings et Prevent duty trainings). Il s'agit de créer une culture bureaucratique nouvelle, sur laquelle la lutte contre la radicalisation puisse venir s'appuyer.

Cette culture s'articule autour d'un certain nombre de valeurs dites britanniques (British values) que le gouvernement entend promouvoir dans le cadre de nombreuses politiques publiques: politiques de la ville, logement, école ou prévention du terrorisme après les attentats de Londres en 2005. La promotion de ces valeurs communes (shared values) s'est rapidement traduite par des mesures concrètes telle que 
l'instauration, dès 2002, d'un serment d'allégeance pour les candidat.e.s à la naturalisation. La procédure de naturalisation comprend désormais un cours sur l'histoire et les valeurs britanniques et est sanctionnée d'un test sur la maîtrise de la langue et des valeurs britanniques. Depuis 2002, les programmes scolaires comprennent des cours de citoyenneté (citizenship education) qui ne sont pas sans rappeler l'enseignement moral et civique (EMC) du système scolaire français. Tout comportement qui saperait ces valeurs devient dans cette perspective un signe de radicalisation et doit à ce titre être signalé par les agent.e.s qui sont en contact avec le public dans le cadre de leurs activités professionnelles. L'« extrémisme» est ainsi défini dans la stratégie Prevent 2011: "vocal or active opposition to fundamental British values, including democracy, the rule of law, individual liberty and mutual respect and tolerance of different faiths and beliefs. We also include in our definition of extremism calls for the death of members of our armed forces $\aleph^{42}$. Les signalements peuvent mener au déclenchement d'une procédure Channel ${ }^{43}$, selon l'évaluation des cas rapportés faite par les services de police. En théorie, elle n'est enclenchée que s'il existe une "vulnérabilité véritable » (genuine vulnerability). Channel n'existe qu'en Angleterre et au Pays de Galles, son équivalent écossais est le Prevent Professional Concern (PPC).

Les indices des "comportements musulmans" qui sont considérés comme déviants peuvent être décelés dans l'extrait d'entretien suivant, réalisé avec une élue, ancienne chargée de mission lutte contre les discriminations dans le secondaire. Récemment élue, elle a fait l'expérience des dernières versions de Prevent au cours de sa vie professionnelle :

The Ofsted inspection of schools says they want to see that you've got Prevent in your safeguarding policies and that you've got to report anybody if you think they are at risk of radicalisation. You've got to have British values in your curriculum. [...] I was working in a sixth form college. I was responsible for safeguarding. You'd have a training around Prevent and the very next day you'd have teachers coming in saying $X$ has written an essay about such and such and there'd be a quote from the Koran in it. They'd say they must be radicalising. I'd say: what are you talking about? [...] They'd say things like that, that it was a risk of radicalisation. And not really understanding at all. [...] And it worried me, because it only just happened when I was in the role I was in. I had a knowledge of Islam and issues of Muslims. If somebody else had been in my role, I don't quite know whether people would have been reported $[. . .]^{44}$.

Cet extrait permet de comprendre d'une part que la politique de prévention de la radicalisation a déjà des conséquences sur les perceptions des comportements musulmans dans l'espace public. En réalité, le contenu des valeurs fondamentales britanniques importe peu. Les formes les plus orthodoxes ou orthopraxes de religiosité apparaissent comme l'indicateur principal de leur infraction et c'est en tant qu'elles sont des signes de radicalisation qu'elles sont l'objet des formations Prevent.

\section{La compatibilité variable de la culture du signalement avec les diverses fonctions publiques britanniques}

Si l'on prend acte des recherches consacrées à la street level bureaucracy, les agent.e.s de guichet doivent être considéré.e.s comme des coproducteurs/trices de l'action publique, et comme des interprètes des politiques publiques ${ }^{45}$. Aussi, la volonté du gouvernement britannique de faire advenir une nouvelle culture bureaucratique propre à la lutte contre la radicalisation se heurte parfois à des cultures 
professionnelles pré-existantes contradictoires, qui peuvent être en partie le résultat de plusieurs dizaines d'années de multiculturalisme municipal. Cependant, elles ne sont pas identiques d'une fonction publique à l'autre et le terrain d'implantation de l'entreprise étatique est plus ou moins favorable. En Écosse, le syndicat UNISON ${ }^{46}$ est par exemple actif dans la dénonciation du Prevent duty.

Ces professionnel.le.s réagissent donc de diverses manières aux signes de radicalisation qu'il est de leur mission de repérer. Il a déjà été question des comportements contraires aux valeurs britanniques. Il faut y ajouter une religiosité accrue (increased religiosity) et des opinions conservatrices sur un plan religieux ou social (religious or social conservative views). Une religiosité accrue, c'est-à-dire une orthopraxie et une orthodoxie en hausse, sont considérées comme la première étape d'une trajectoire déviante dont l'ultime étape est la perpétration d'actes terroristes. Lors d'une formation WRAP à laquelle nous avons pu assister dans les bâtiments de la mairie de Bristol, il est demandé aux participant.e.s de réfléchir en groupes aux motifs de préoccupation qui peuvent justifier un signalement. Les différents groupes dressent à peu près la liste suivante : « withdrawing or engaging with a new group; assertive behaviour; one track in conversation; language change; change in appearange; change of routine; tatoos; showing anger; being passionate about something; blame culture; comitting criminal acts; becoming obsessive; becoming distant from friends and family; increased use of the Internet ${ }^{47}$. Sauf exception, les signes auxquels doivent porter attention les professionnel.le.s ne sont en rien des comportements criminels mais des opinions et des attitudes. Il s'agit là d'une des conséquences de l'évolution controversée de Prevent vers la lutte contre l'extrémisme tout court plutôt que contre l'extrémisme violent ${ }^{48}$.

31 Le processus d'étiquetage qui fait des pratiques rituelles musulmanes des pratiques déviantes s'appuie sur les outils que sont les formations WRAP ou Prevent duty en tant qu'elles sont des espaces où s'élaborent les normes sociales considérées enfreintes. Lorsque les agent.e.s auxquel.le.s s'adressent ces formations adoptent un regard critique vis-à-vis de leur contenu, ils et elles pointent notamment du doigt la contradiction entre Prevent duty et Equality duty, qui interdit aux administrations publiques de discriminer sur la base d'opinions religieuses ou politiques exprimées par les bénéficiaires ${ }^{49}$. La focalisation de facto de Prevent sur les populations musulmanes donne la sensation aux agent.e.s de devoir appliquer une législation d'exception, qui ne s'applique pas aux autres minorités religieuses ou ethniques. On trouve ce type de mise à distance dans les propos des participant.e.s à une formation à Prevent qui s'est tenue à Bristol en mai 2017. Issu.e.s de divers secteurs professionnels (enseignement et formation, économie sociale et solidaire, conseil pénitentiaire en insertion et probation), ils et elles sont globalement d'accord pour penser que les valeurs britanniques vis-à-vis desquelles il faut évaluer le comportement des usager.e.s conduisent à « stéréotyper » et qu'il s'agit d'une politique conservatrice au sens où elle a été élaborée par le parti conservateur. En réalité, il convient de rappeler que la législation anti-terroriste est d'abord le résultat de décisions prises par des gouvernements travaillistes, en particulier au lendemain des attentats de Londres. Les gouvernements conservateurs qui leur ont succédé ont pu s'appuyer sur cette base pour renforcer le dispositif, notamment à la suite des attentats de l'année 2017, comme celui de Manchester. Il est perceptible que les professionnel.le.s, qui participent ce jour-là à la formation, ont été socialisé.e.s par leur formation ou au cours de leur exercice professionnel.le.s à ce qui s'appelle encore le multiculturalisme et en tous les 
cas à la lutte contre les discriminations en ce qu'elle implique de prendre en compte la diversité des pratiques culturelles dans la manière de fournir les services publics ${ }^{50}$.

La remise en cause de Prevent et la résistance à son application par la fonction publique sont particulièrement fortes et organisées en Écosse. Le collectif SACC mentionné cidessus participe par exemple en mars 2016 à une journée de réflexion sur la lutte contre l'extrêmisme organisée par le Muslim Council of Scotland dans les locaux de la mairie de Glasgow. Étaient notamment présents le député (Member of Scottish Parliament) et ministre de la Justice (Cabinet Secretary for Justice), un conseiller municipal, Archie Graham et Franck Deas, employé de la mairie de Glasgow en charge du suivi du Glasgow Community Planning Partnership et en particulier du Safe Glasgow Group, dont les prérogatives incluent la mise en application de Prevent à Glasgow. Les trois hommes sont interrogés quant à la mise en œuvre de Prevent en Écosse en dépit de son caractère raciste et islamophobe dénoncé par une partie du secteur associatif et syndical. Un tract distribué à l'occasion de réunions publiques et que nous avons découvert lors d'un événement intitulé "Let's Talk About Hate» qui s'est tenu à Edinburgh le 26 juillet 2017, contient les éléments suivants : "When Muslims engage in perfectly normal behaviour such as questioning the status quo or disagreeing with UK foreign policy this is seen as a step on the road to terrorism 》 ou encore : "Prevent feeds racism and Islamophobia by suggesting that legal, peaceful behaviour when pursued by Muslims is a cause for concern and police intervention ». L'annonce faite par Archie Graham : "most teachers would be getting Prevent training at the end of the summer holidays ", n'est pas de nature à les rassurer en dépit de l'insistance du conseiller: "the changes in an individual's behaviour that might trigger PPC were not necessarily specifically associated with devotion to Islam $"{ }^{51}$. Outre SACC et le Muslim Council of Britain, la journée d'étude compte également sur la participation de l'association Islamic Society of Britain, de la Federation of Student Islamic Societies (FOSIS) et le centre de recherche sur l'Islam contemporain de l'Université d'Edinburgh, Al Waleed.

Les agent.e.s peuvent d'autre part s'étonner de la focalisation du contenu des formations sur l'islam afin de construire une critique des politiques de lutte contre la radicalisation. Cette critique est très ancienne et elle existait déjà dès la première version du programme Prevent. C'est d'ailleurs la raison pour laquelle l'extrême droite est aujourd'hui incluse parmi les extrémismes que le gouvernement britannique se donne pour objectif de combattre ${ }^{52}$. Lors de la formation aux obligations Prevent à laquelle nous avons assistée en avril 2018, six groupes sont considérés comme extrémistes: les islamistes, l'extrême droite, les militant.e.s pour les droits des animaux, les nationalistes, les militant.e.s anti-avortement, certain.e.s indépendantistes nord-irlandais.e.s (celles et ceux prenant part à la lutte armée) ${ }^{53}$. Il existe donc une capacité de résistance certaine des agent.e.s de la fonction publique britannique au traitement d'exception réservé aux populations musulmanes et les évolutions du programme Prevent depuis son introduction en constituent un indice.

\section{Conclusion}

Malgré deux modèles nationaux de régulation du fait religieux, la comparaison francobritannique qui prend appui sur une analyse en termes de processus d'étiquetage fait apparaître des formes de convergence récentes entre le gouvernement des pratiques musulmanes en France et au Royaume-Uni. À travers la restitution de matériaux issus 
de quatre terrains d'enquête, nous avons montré que la laïcité en tant qu'elle sert de prétexte à la mise en discussion des pratiques musulmanes ainsi que la politique de lutte contre la radicalisation au Royaume-Uni aboutissent l'une et l'autre à l'exceptionnalisation des signes extérieurs d'islamité dans l'espace public et à l'adoption de législations et de règles qui tendent à normaliser l'exception. Qu'ils soient jugés à l'aune de leur neutralité ou des valeurs britanniques fondamentales, ces comportements sont constitués en infractions de normes sociales et étiquetés comme déviants. Cet étiquetage justifie des mesures de contrôle voire d'interdiction dont la mise en œuvre est confiée à des groupes sociaux de plus en plus larges. En France, ce traitement à part de l'islam passe tout d'abord par sa mise en discussion au travers du principe général laïque. Ce principe fait néanmoins l'objet de luttes définitionnelles. Certaines interprétations de la laïcité mettent l'accent sur la neutralité, ce qui peut conduire à une nouvelle définition des frontières de l'espace public et ainsi à l'exclusion de signes extérieurs de religiosité, en particulier musulmans, et à la disciplinarisation des comportements musulmans hors de la sphère privée. Un processus similaire peut être observé au Royaume-Uni mais son origine est plus clairement gouvernementale et tient à la conjonction entre le renforcement progressif des politiques de lutte contre la radicalisation et le changement de référentiel de politiques publiques à travers la community cohesion qui met l'accent sur le partage de valeurs communes. Néanmoins, le basculement de culture bureaucratique ne semble pas être effectif outre-Manche et les contradictions entre la politique de lutte contre les discriminations et la législation anti-terroriste constitue le socle d'une résistance de la part des professionnel.le.s et bénévoles de terrain.

Que l'impulsion soit étatique dans un cas ou qu'elle relève davantage de l'action collective, le résultat est similaire. Malgré les résistances qui s'expriment parfois, les opinions et attitudes musulmanes sont scrutées de manière croissante, comme si l'action publique s'adaptait de cette manière à l'individualisation et à la subjectivisation du croire ${ }^{54}$ et devait pour ce faire se déployer au plus près des corps et des consciences musulmanes. Malgré l'énonciation de l'action publique en des termes génériques, le fait qu'elle s'adresse implicitement aux populations musulmanes suscite souvent la contestation d'une entreprise qui les fait sortir du droit commun pour les faire entrer dans le domaine de l'exception.

\section{BIBLIOGRAPHIE}

AMIRAUX Valérie, « Après le 7 janvier 2015, quelle place pour le citoyen musulman en contexte libéral sécularisé ?», Multitudes, n 59/2, 2015, p. 83-93.

ARÈNES Claire, La prévention du terrorisme en Grande-Bretagne : le programme Prevent et la communauté musulmane, Paris, PUF, 2016.

BARRAS Amélie, Refashioning secularisms in France and Turkey. The case of the headscarf ban, London, Routledge, « Islamic Studies Series », 2014. 
BAUBÉROT Jean, Les sept laïcités françaises : le modèle français de laïcité n'existe pas, Paris, Éditions de la Maison des sciences de l'Homme, 2015.

BECKER Howard, Outsiders: studies in the sociology of deviance, New York, The Free Press, 1963.

BLANC Nicolas, «Le niqab devant les tribunaux canadiens et la circulation occidentale du corps islamique ", Les Cahiers de la Justice, $\mathrm{n}^{\circ} 3 / 3,2014$, p. 463-479.

COMITÉ CONSUlTATF LAḮcITÉ, Pour une Charte Rennaise de la Laïcité, Ville de Rennes, 2016

CROWLEY John, « Usages de la gouvernance et de la gouvernementalité », Critique internationale, $n^{\circ} 4 / 21,2003$, p. 52-61.

DIRECTION ENFANCE ÉDUCATION, Référentiel commun autour de l'application du principe de laïcité sur les temps péri et extra scolaires à la Ville de Rennes, Ville de Rennes, 2017.

GARBAYE Romain, « Le New Labour et la community cohesion. Émergence et échec d'une approche de la diversité », in COHEN James et al., L'Atlantique multiraciale, Editions Karthala, 2012, p. 163-180.

GöLE Nilufer, Musulmans au quotidien : une enquête européenne sur les controverses autour de l'Islam, Paris, La Découverte, 2015.

GUSFIELD Joseph, Symbolic Crusade. Status Politics and the American Temperance Movement, Urbana and Chicago, University of Illinois Press, 1986 ( $2^{\mathrm{e}}$ édition).

HENNETTE-VAUCHEZ Stéphanie, « Séparation, garantie, neutralité... les multiples grammaires de la laïcité ", Les Nouveaux Cahiers du Conseil constitutionnel, n53/4, 2016, p. 9-19.

HENNETTE-VAUCHEZ Stéphanie et VALENTIN Vincent, L'affaire Baby Loup ou La nouvelle laïcité, Issy-lesMoulineaux, Lextenso éditions, 2014.

HERVIEU-LÉGER Danièle, Le pélerin et le converti. La religion en mouvement, Paris, Flammarion, 1999.

HM GOVERNMENT, Revised Prevent Duty Guidance: for England and Wales, 2015.

HM GOVERNMENT, CONTEST. The United Kingdom's Strategy for Countering Terrorism, 2018.

INNES Martin, ROBERTS Colin, et LOWE Trudy, « A disruptive influence? "Preventing" problems and countering violent extremism policy in practice ", Law and society review, $\mathrm{n}^{\circ} 51 / 2,2017$,

p. 252-281.

HUPE Peter, BUFFAT Aurélien, et HILL Michael (dir.), Understanding street-level bureaucracy, Bristol, Policy Press, 2015.

KUNDNANI Arun, Spooked! How not to prevent violent extremism, Institute of Race Relations, 2009.

LAMINE Anne-Sophie (dir.), Quand le religieux fait conflit. Désaccords, négociations ou arrangements, Rennes, PUR, «Sciences des religions », 2013.

LORCERIE Françoise, « Usages publics de l'islam en France. Entre altérité et établissement », in LAMINE Anne-Sophie (dir.), Quand le religieux fait conflit. Désaccords, négociations ou arrangements, Rennes, PUR, « Sciences des religions », 2013.

LORCERIE Françoise (dir.), La politisation du voile : l'affaire en France, en Europe et dans le monde arabe, Paris, L'Harmattan, 2005.

MOHAMMED Jahangir, « Britain's Counter Extremism Policies are Criminalising Muslim Thought and Expression ", Public Spirit [En ligne], 27 octobre 2015, consulté le 23 décembre 2019. URL : http://www.publicspirit.org.uk/britains-counter-extremismpolicies-are-criminalising-muslimthought-and-expression/. 
NEVEU Érik, Sociologie des mouvements sociaux, Paris, La Découverte, 2005.

O'TOOLE Therese, LEWICKI Aleksandra, et MODOOD Tariq, Building the Bridge: Muslim community engagement in Bristol, Bristol, Centre for the study of ethnicity and citizenship, 2014.

PINGAUD Étienne, L'implantation de l'islam dans les « quartiers ». Contribution à l'analyse du succès d'une offre symbolique, Thèse de doctorat de sociologie, École des hautes études en sciences sociales, sous la direction de MAUGER Gérard, Paris, 2013.

SACC, "Muslim Council of Scotland Looks at Counter-Extremism Policy », Scotland Against Criminalising Communities, 8 mars 2016, consulté le 6 mai 2020. URL : https://www.sacc.org.uk/ news/2016/muslim-council-scotland-looks-counter-extremism-policy

SACC, « What is Prevent », Scotland Against Criminalising Communities, 27 avril 2017, consulté le 6 mai 2020. URL : https://www.sacc.org.uk/articles/2017/what-prevent SANNA Maria Eleonora, «Ces corps qui ne comptent pas : les musulmanes voilées en France et au Royaume-Uni », Cahiers du Genre, n 1/50, 2011, p. 111-132.

WELCH Michael, «Foreword "», in PoynTING Scott et MORGAN George (dir.), Global Islamophobia. Muslims and moral panic in the West, Farnham, Ashgate, 2012, p. ix-xii.

«Une cérémonie de vœux en forme d'hommage aux victimes », Ouest-France, 9 janvier 2015, p. Nantes Métropole.

\section{NOTES}

1. Laïque n'est pas l'équivalent de secular dans la mesure où le terme désigne la séparation des cultes et de la sphère publique, quelle que soit la forme prise par cette séparation. La sécularisation désigne quant à elle un phénomène de recul des croyances religieuses et/ou de l'emprise des institutions religieuses sur la vie sociale.

2. Cette entreprise, avant même d'être tournée vers l'extérieur du mouvement laïque, consiste en une lutte interne pour l'imposition d'une définition de la laïcité plutôt qu'une autre. L'hétérogénéité du mouvement laïque n'est pas l'objet de cet article mais elle ne doit pas être sous-estimée et mérite une analyse ad hoc.

3. BARRAS Amélie, Refashioning secularisms in France and Turkey. The case of the headscarf ban, London, Routledge, « Islamic Studies Series », 2014, p. 43.

4. Ibid., p. 53.

5. WELCH Michael, "Foreword», in POYNTING Scott et MORGAN George (dir.), Global Islamophobia. Muslims and moral panic in the West, Farnham, Ashgate, 2012, p. ix.

6. GÖLE Nilufer, Musulmans au quotidien : une enquête européenne sur les controverses autour de l'Islam, Paris, La Découverte, 2015, p. 20.

7. BECKER Howard, Outsiders: studies in the sociology of deviance, New York, The Free Press, 1963.

8. GUSFIELD Joseph, Symbolic Crusade. Status Politics and the American Temperance Movement,Urbana and Chicago, University of Illinois Press, 1986 ( $2^{\mathrm{e}}$ édition).

9. Quoique Becker et Gusfield emploient tous deux le terme « moral(e) », nous privilégions pour notre part l'usage du terme "symbolique », moins connoté négativement et parce qu'il renvoie ici à des mobilisations qui visent l'application de règles ou l'obtention de ressources qui ne sont pas économiques et matérielles. Ces règles et ressources relèvent des domaines cognitif, normatif et axiologique.

10. Deux centres culturels islamiques, un centre culturel juif et un centre culturel bouddhiste. 
11. вАUвÉROT Jean, Les sept laïcités françaises : le modèle français de laïcité n'existe pas, Paris, Éditions de la Maison des sciences de l'Homme, 2015. La typologie de Jean Baubérot distingue sept formes de laïcité. Quatre de ces laïcités sont historiques : la laïcité antireligieuse, la laïcité gallicane et les deux laïcités libérales ou séparatistes dans leurs conceptions individualiste ou collective. Trois laïcités sont plus récentes, il s'agit de la laïcité « ouverte », de la laïcité identitaire et de la laïcité alsacienne-mosellane.

12. NEVEu Érik, Sociologie des mouvements sociaux, Paris, La Découverte, 2005.

13. À savoir l'Association régionale des œuvres éducatives et de vacances de l'Education nationale (Aroéven), l'association Égalité, laïcité, Europe (ÉGALE), le Cercle Paul Bert, le Comité laïque Rennais, les Délégués départementaux de l'Éducation nationale (DDEN), la Fédération des conseils de parents d'élèves (FCPE), la Fédération syndicale unitaire (FSU) 35, la Ligue des droits de l'homme (LDH), la Ligue de l'enseignement, le Syndicat national unitaire des instituteurs, professeurs des écoles et PEGC (SNUipp 35), l'Union des familles laïques (UFAL), l'Union des associations interculturelles rennaises (UAIR), la Maison internationale de Rennes (MIR).

14. LAMINE Anne-Sophie (dir.), Quand le religieux fait conflit. Désaccords, négociations ou arrangements, Rennes, PUR, «Sciences des religions », 2013, p. 8.

15. LORCERIE Françoise, « Usages publics de l'islam en France. Entre altérité et établissement », in LAMINE Anne-Sophie (dir.), Quand le religieux fait conflit. Désaccords, négociations ou arrangements, Rennes, PUR, « Sciences des religions », 2013, p. 15.

16. Notes de terrain, réalisé à Rennes lors de formations à la laïcité le 4 octobre 2016

17. HENNETTE-VAUCHEZ Stéphanie et VALENTIN Vincent, L'affaire Baby Loup ou La nouvelle laïcité, Issyles-Moulineaux, Lextenso éditions, 2014.

18. AMIRAUX Valérie, « Après le 7 janvier 2015, quelle place pour le citoyen musulman en contexte libéral sécularisé ?", Multitudes, n 59/2, 2015, p. 89.

19. BARRAS, op. cit., p. 59.

20. comité ConsultatF LAïcité, Pour une Charte Rennaise de la Laïcité, Ville de Rennes, 2016, p. 12.

21. HENNETTE-VAUCHEZ Stéphanie, « Séparation, garantie, neutralité... les multiples grammaires de la laïcité ", Les Nouveaux Cahiers du Conseil constitutionnel, n 53/4, 2016, p. 9-19.

22. "Une cérémonie de vœux en forme d'hommage aux victimes ", Ouest-France, 9 janvier 2015, p. Nantes Métropole

23. Le terme " ostentatoire » est utilisé au cours de ces échanges. Toutefois, rappelons que la loi du 15 mars 2004 est relative au "port de signes ou de tenues manifestant une appartenance religieuse » et que son article L. 141-5-1 mentionne « le port de signes ou tenues par lesquels les élèves manifestent ostensiblement une appartenance religieuse ». Le glissement sémantique n'est pas anodin.

24. Notes de terrain, réalisé à Rennes le 11 octobre 2016.

25. LORCERIE Françoise (dir.), La politisation du voile: l'affaire en France, en Europe et dans le monde arabe, Paris, L'Harmattan, 2005.

26. DIRECTION ENFANCE ÉDUCATION, Référentiel commun autour de l'application du principe de laïcité sur les temps péri et extra scolaires à la Ville de Rennes, Ville de Rennes, 2017, p. 33

27. Entretien avec Asma B., salariée du CID, réalisé à Nantes le 15 juin 2016.

28. Cette notion foucaldienne renvoie à deux dimensions : la disciplinarisation des corps et la gestion des populations par le pouvoir étatique (par exemple la démographie en tant que science de l'État). L'usage du terme implique l'adoption d'une perspective critique vis-à-vis des processus de gouvernement. On peut se référer à l'article de John Crowley sur les usages de la gouvernance et de la gouvernementalité ou aux articles de Nicolas Blanc et de Maria Eleonora Sanna pour deux illustrations de la gestion des corps musulmans par les puissances publiques occidentales: CROWLEY John, "Usages de la gouvernance et de la gouvernementalité », Critique internationale, $\mathrm{n}^{\circ} 4 / 21,2003$, p. 52-61 ; BLANC Nicolas, « Le niqab devant les tribunaux canadiens et la circulation 
occidentale du corps islamique », Les Cahiers de la Justice, n³/3, 2014, p. 463-479; sANNA Maria Eleonora, «Ces corps qui ne comptent pas : les musulmanes voilées en France et au Royaume-Uni », Cahiers du Genre, $\mathrm{n}^{\circ}$ 1/50, 2011, p. 111-132.

29. Sur la base d'informations fournies par les services de renseignements et non plus sur une base démographique.

30. O'TOOLE Therese, LEWICKI Aleksandra, et MODOOD Tariq, Building the Bridge: Muslim community engagement in Bristol, Bristol, Centre for the study of ethnicity and citizenship, 2014.

31. KUNDNANI Arun, Spooked! How not to prevent violent extremism, Institute of Race Relations, 2009.

32. Ibid. p. 18.

33. GARBAYE Romain, «Le New Labour et la community cohesion. Émergence et échec d'une approche de la diversité ", in coHEN James et al., L'Atlantique multiraciale, Editions Karthala, 2012, p. $163-180$.

34. Après 2011, la gestion de Prevent est entièrement confiée à l'Office for Security and CounterTerrorism (OSCT) du Home Office et non plus partagée avec le Department for Communities and Local Government (DCLG).

35. HM GOVERNMENT, CONTEST. The United Kingdom's Strategy for Countering Terrorism, 2018.

36. HM GOVERNMENT, Revised Prevent Duty Guidance: for England and Wales, 2015, p. 10.

37. HM GOVERNMENT, op. cit., p. 35.

38. SACC, "What is Prevent ", Scotland Against Criminalising Communities, 27 avril 2017, consulté le 6 mai 2020. URL : https://www.sacc.org.uk/articles/2017/what-prevent

39. Notes de terrain, réalisé à Bristol lors d'un atelier WRAP le 21 mars 2017.

40. Entretien avec Saqib V., réalisé à Bristol en avril 2017.

41. ARÈNES Claire, La prévention du terrorisme en Grande-Bretagne: le programme Prevent et la communauté musulmane, Paris, PUF, 2016, p. 100.

42. HM GOVERNMENT, op. cit., p. 4.

43. Programme de prise en charge des signalements pour radicalisation.

44. Entretien avec Rose Q., réalisé à Bristol en février 2017.

45. HUPE Peter, BUFFAT Aurélien, et HILL Michael (dir.), Understanding street-level bureaucracy, Bristol, Policy Press, 2015.

46. UNISON est le principal syndicat de la fonction publique britannique. Il rassemble 1,3 million de syndiqué.e.s, dont 150000 en Écosse.

47. Notes de terrain, réalisé à Bristol lors d'un atelier WRAP le 21 mars 2017.

48. INNES Martin, ROBERTS Colin, et LOWE Trudy, «A disruptive influence? "Preventing" problems and countering violent extremism policy in practice ", Law and society review, $n^{\circ} 51 / 2,2017$, p. 252-281.

49. MOHAMMED Jahangir, « Britain's Counter Extremism Policies are Criminalising Muslim Thought and Expression », Public Spirit [En ligne], 27 octobre 2015, consulté le 23 décembre 2019. URL : http://www.publicspirit.org.uk/britains-counter-extremismpolicies-are-criminalising-muslimthought-and-expression/.

50. Notes de terrain, réalisé à Bristol d'une formation au Prevent Duty, le 23 mai 2017.

51. SACC, "Muslim Council of Scotland Looks at Counter-Extremism Policy », Scotland Against Criminalising Communities, 8 mars 2016, consulté le 6 mai 2020. URL : https://www.sacc.org.uk/ news/2016/muslim-council-scotland-looks-counter-extremism-policy

52. HM GOVERNMENT, op. cit.

53. Notes de terrain, réalisé à Bristol lors d'une formation au Prevent Duty le 17 avril 2018.

54. HERVIEU-LÉGER Danièle, Le pélerin et le converti. La religion en mouvement, Paris, Flammarion, 1999. 


\section{RÉSUMÉS}

L'étude de la gouvernance du fait musulman par quatre municipalités françaises et britanniques révèle des convergences de part et d'autre de la Manche, malgré des modèles nationaux souvent renvoyés dos à dos. Qu'il s'agisse de la mobilisation d'une partie du secteur associatif laïque à Rennes et à Nantes ou de la recentralisation de la gestion du programme de lutte de la radicalisation Prevent par le gouvernement britannique, les deux phénomènes aboutissent à une disciplinarisation des comportements musulmans dans l'espace public. Dans les quatre cas d'étude, et en dépit de résistances d'amplitudes variables, certain.e.s acteurs et actrices de terrain, professionnel.le.s ou bénévoles, sont invité.e.s par les autorités municipales ou gouvernementales à envisager l'orthodoxie et l'orthopraxie musulmanes comme des comportements déviants vis-à-vis des normes séculières et parfois même comme des signes de radicalisation qui menacent l'ordre public et qui méritent donc un traitement d'exception.

The study of the governance of Islam in four cities in France and in the United Kingdom reveals convergences on both sides of the Channel, despite national models that are often presented at odds with each other. Whether it be the mobilisation by some part of the laïque ${ }^{1}$ voluntary sector in Rennes and Nantes or the recentralisation of the governance of the counter-extremism policy Prevent by the British government, both phenomena result in the disciplinary government of Muslim conduct in public spaces. In the four case studies, and in spite of various degrees of resistance, grassroots players, both professionals and volunteers, are encouraged by city and government authorities to consider Muslim orthodoxy and orthopraxis as deviant conduct with regard to secular norms, and in some cases even as signs of radicalisation that pose a threat to public order and therefore call for exceptional measures.

\section{INDEX}

Mots-clés : fait musulman, laïcité, radicalisation, disciplinarisation, étiquetage, espace public

Keywords : Islam, secularism, radicalisation, disciplinary government, labelling, public spaces

\section{AUTEUR}

\section{ALICE PICARD}

Alice Picard est docteure en science politique de l'Université de Rennes 1 et membre du laboratoire Arènes (UMR 6051). Sa thèse porte sur la gouvernance du fait musulman dans quatre municipalités françaises et britanniques et s'intéresse à la reconfiguration des frontières symboliques que la visibilité de la religion musulmane dans les espaces publics en France et au Royaume-Uni engendre.

Alice Picard, Arènes, UMR 6051, Université de Rennes 1, 35700, Rennes 